\title{
DISCOVERY OF INTERSTELLAR HEAVY WATER
}

\author{
H. M. Butner, ${ }^{1,2}$ S. B. Charnley, ${ }^{3}$ C. Ceccarelli, ${ }^{4}$ S. D. Rodgers, ${ }^{3}$ J. R. Pardo, ${ }^{5}$ \\ B. Parise, ${ }^{6}$ J. Cernicharo, ${ }^{5}$ and G. R. Davis \\ Received 2006 November 5; accepted 2007 March 7; published 2007 March 21
}

\begin{abstract}
We report the discovery of doubly deuterated water $\left(\mathrm{D}_{2} \mathrm{O}\right.$, heavy water) in the interstellar medium. Using the James Clerk Maxwell Telescope and the Caltech Submillimeter Observatory $10 \mathrm{~m}$ telescope, we detected the $1_{10}-1_{01}$ transition of para- $\mathrm{D}_{2} \mathrm{O}$ at $316.7998 \mathrm{GHz}$ in both absorption and emission toward the protostellar binary system IRAS $16293-2422$. Assuming that the $\mathrm{D}_{2} \mathrm{O}$ exists primarily in the warm regions where water ices have been evaporated (i.e., in a "hot corino" environment), we determine a total column density of $N\left(\mathrm{D}_{2} \mathrm{O}\right)$ of $1.0 \times 10^{13} \mathrm{~cm}^{-2}$ and a fractional abundance of $\mathrm{D}_{2} \mathrm{O} / \mathrm{H}_{2}=1.7 \times 10^{-10}$. The derived column density ratios for IRAS $16293-2422$ are $\mathrm{D}_{2} \mathrm{O} / \mathrm{HDO}=1.7 \times 10^{-3}$ and $\mathrm{D}_{2} \mathrm{O} / \mathrm{H}_{2} \mathrm{O}=5 \times 10^{-5}$ for the hot corino gas. Steady state models of water ice formation, either in the gas phase or on grains, predict $\mathrm{D}_{2} \mathrm{O} / \mathrm{HDO}$ ratios that are about 4 times larger than that derived from our observations. For water formation on grain surfaces to be a viable explanation, a larger $\mathrm{H}_{2} \mathrm{O}$ abundance than that measured in IRAS 16293-2422 is required. Alternatively, the observed $\mathrm{D}_{2} \mathrm{O} / \mathrm{HDO}$ ratio could be indicative of gas-phase water chemistry prior to a chemical steady state being attained, such as would have occurred during the formation of this source. Future observations with the Herschel Space Observatory satellite will be important for settling this issue.
\end{abstract}

Subject headings: astrochemistry — ISM: individual (IRAS 16293-2422) — ISM: molecules — stars: formation

\section{INTRODUCTION}

It has long been known that the cold temperatures found in dense molecular clouds ( $10-30 \mathrm{~K}$ ) would be conducive to the selective incorporation of deuterium atoms into interstellar molecules (see Watson 1976 and references therein). At $10 \mathrm{~K}$, the zero-point energy difference between $\mathrm{H}_{2} \mathrm{D}^{+}$and $\mathrm{H}_{3}^{+}$leads to the former ion becoming sufficiently abundant that large numbers of $\mathrm{D}$ atoms can be distributed to other molecules through ion-molecule exchange reactions (e.g., Millar et al. 1989). The abundance of deuterated molecules can be significantly enhanced (to a few percent of the common variant), compared to the much smaller Galactic D/H ratio $\left(\sim 1.5 \times 10^{-5}\right)$. The main reservoir is $\mathrm{HD}$, but electron dissociative recombination of $\mathrm{H}_{2} \mathrm{D}^{+}$leads to enhanced concentrations of free $\mathrm{D}$ atoms in the gas. Catalytic reactions on dust grains, driven by the accretion of $\mathrm{H}$ and $\mathrm{D}$ atoms from the gas, can therefore also produce large D enrichments in interstellar ices (Tielens 1983; Charnley et al. 1997). Deuterium enrichments are found in meteorites and comets, and so interstellar deuteration may have a connection with that of primitive solar system matter (e.g., Nuth et al. 2006).

Over the last few years, observations have found very high $\mathrm{D} / \mathrm{H}$ ratios in some molecules, $\sim 10,000$ times the Galactic value (for reviews, see Ceccarelli 2002; Roueff \& Gerin 2003; Ceccarelli et al. 2007), as well as doubly deuterated and triply deuterated isotopologues of common interstellar molecules, such as

\footnotetext{
Joint Astronomy Centre, University Park, Hilo, HI 96720; h.butner@ jach.hawaii.edu.

${ }^{2}$ Current address: Department of Physics, James Madison University, Harrisonburg, VA 22807-7702.

${ }^{3}$ Space Science and Astrobiology Division, NASA Ames Research Center, Moffett Field, CA 94035.

${ }^{4}$ Laboratoire d'Astrophysique, Observatoire de Grenoble, 38041 Grenoble Cedex 09, France.

${ }^{5}$ Departamento de Astrofisica Molecular e Infrarroja, Instituto de Estructura de la Materia, CSIC, E-28006 Madrid, Spain.

${ }^{6}$ Max Planck Institut für Radioastronomie, 53121 Bonn, Germany.
}

ammonia, hydrogen sulfide, thioformaldehyde, formaldehyde, and methanol (Lis et al. 2002; van der Tak et al. 2002; Vastel et al. 2003, 2004; Marcelino et al. 2005; Parise et al. 2002, 2004). The region containing the low-mass protostellar binary system IRAS 16293-2422 has been particularly well studied in deuterated molecules (van Dishoeck et al. 1995; Ceccarelli et al. 2007). In IRAS 16293-2422, it appears that several of the deuterated molecules (e.g., water and methanol; Parise et al. 2002, 2005) are present in small "hot corinos," the low-mass analogs of the hot molecular cores found in regions of massive star formation, in which icy grain mantles have been evaporated (Cazaux et al. 2003; Kuan et al. 2004; Bottinelli et al. 2004). The origin of this "superdeuteration" lies in gas-grain chemical processes associated with cold prestellar cores. In these dense regions $\left(\gtrsim 10^{6} \mathrm{~cm}^{-3}\right)$, as $\mathrm{CO}$ molecules are lost from the gas through condensation onto dust (e.g., Bacmann et al. 2003), the abundances of $\mathrm{H}_{2} \mathrm{D}^{+}, \mathrm{HD}_{2}^{+}, \mathrm{D}_{3}^{+}$, and atomic $\mathrm{D}$ all increase dramatically and drive the fractionation chemistry to produce greatly elevated molecular D/H ratios (Roberts et al. 2003).

Comparison of the $\mathrm{HDO} / \mathrm{H}_{2} \mathrm{O}$ ratios of comets with that of ocean water and with interstellar ratios can provide important cosmogonic information (Meier et al. 1998; Bockelée-Morvan et al. 1998). In moving toward a complete understanding of interstellar deuterium chemistry, the detection of several key isotopologues will be required. An important, yet puzzling, aspect of interstellar deuteration concerns the markedly low levels of deuterium fractionation commonly measured in water, especially when compared to other molecules. The lower $\mathrm{HDO} / \mathrm{H}_{2} \mathrm{O}$ ratios $\left(\sim 3 \times 10^{-4}\right)$ measured in massive hot molecular cores (e.g., Jacq et al. 1990; Gensheimer et al. 1996) are consistent with ion-molecule chemistry at around $30 \mathrm{~K}$ (Millar et al. 1989), whereas the highest ratios (0.004-0.01) are more suggestive of grain-surface reactions (Pardo et al. 2001). Recently, it has become possible to compare solar system $\mathrm{D} / \mathrm{H}$ ratios with those of more primitive analog systems. In the protostellar binary system IRAS 16293-2422, the bounds on the measured range of $\mathrm{HDO} / \mathrm{H}_{2} \mathrm{O}$ ratios $\left(2 \times 10^{-4}\right.$ to $3 \times 10^{-2}$; Stark et al. 2004; Parise 
TABLE 1

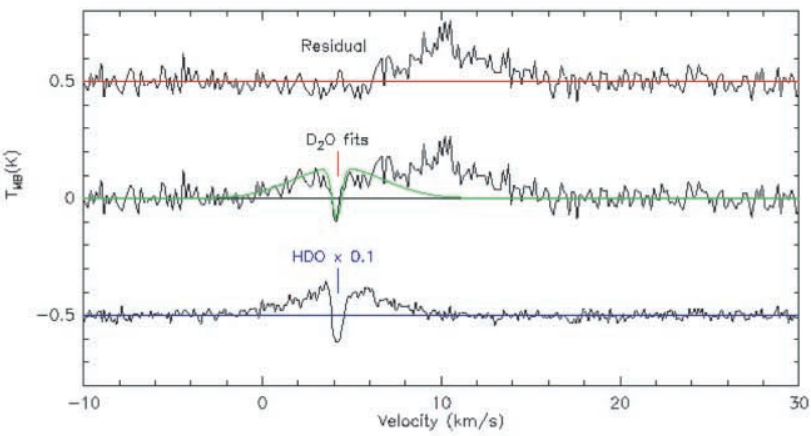

FIG. 1.-The $1_{10}-1_{01}$ transition of para- $\mathrm{D}_{2} \mathrm{O}$ at $316.7998 \mathrm{GHz}$ detected in emission and in absorption toward I16293A (center). A two-Gaussian component fit (green), using the velocity width and $V_{\mathrm{LSR}}$ of the HDO line reported by Stark et al. (2004), is superimposed on the $\mathrm{D}_{2} \mathrm{O}$ spectrum. This HDO line (scaled by $\times 0.1$ ) is shown at bottom and, on top, an emission component is evident in the residual spectra obtained after the $\mathrm{D}_{2} \mathrm{O}$ Gaussians have been subtracted.

et al. 2005) are determined by the abundances measured in the cold extended envelope and in the hot corino. Furthermore, Ceccarelli et al. (2005) have detected HDO in the DM Tau protoplanetary disk and have argued that photodesorption of water ices could lead to $\mathrm{HDO} / \mathrm{H}_{2} \mathrm{O} \geqslant 0.01$ in the outer disk gas (Ceccarelli \& Dominik 2005).

Interstellar water could be formed by three distinct chemical routes, and the deuterium fractionation expected from each has been evaluated: hydrogenation of oxygen atoms on cold dust grains (e.g., Caselli et al. 2002), ion-molecule reactions in cold gas (e.g., Millar et al. 1989), and neutral-neutral reactions in hot shocked gas (Bergin et al. 1999). Observations of multiply deuterated molecules could in principle distinguish between gasphase or grain-surface deuteration pathways (Rodgers \& Charnley 2001). In the case of $\mathrm{H}_{2} \mathrm{O}$, measuring the abundance of heavy water $\left(\mathrm{D}_{2} \mathrm{O}\right)$ could shed light on both the origin of water and the evolution of molecular clouds (Rodgers \& Charnley 2002). In this Letter, we report the discovery of interstellar heavy water in absorption toward IRAS 16293-2422.

\section{OBSERVATIONS}

We observed the para- $\mathrm{D}_{2} \mathrm{O}\left(1_{10}-1_{01}\right)$ fundamental transition at $316.799810 \mathrm{GHz}$ using the James Clerk Maxwell Telescope $(\mathrm{JCMT})^{7}$ in the direction of IRAS $16293-2422$, at the nominal position $\alpha=16^{\mathrm{h}} 32^{\mathrm{m}} 22.9^{\mathrm{s}}, \delta=-24^{\circ} 28^{\prime} 36.0^{\prime \prime}$ (J2000.0), which coincides with the A component of the binary system forming IRAS 16293-2422. The observations were obtained between 2005 March and July. The line was observed with the $345 \mathrm{GHz}$ SIS receiver RxB3 in dual-channel single-sideband (SSB) mode using the lower sideband. Typical SSB temperatures were between 550 and $750 \mathrm{~K}$. The observations were made in positionswitched mode using a reference position $800^{\prime \prime}$ east of IRAS 16293-2022 with frequent pointing checks using the standard JCMT continuum position for IRAS 16293-2422. The Digitial Autocorrelator Spectrometer back end was split into two sections of $125 \mathrm{MHz}$ with a spectral resolution of $156 \mathrm{kHz}$, corresponding to an effective resolution of $0.18 \mathrm{~km} \mathrm{~s}^{-1}$ at $316.8 \mathrm{GHz}$. At $316.8 \mathrm{GHz}$, the FWHM beamwidth of the JCMT is about $15^{\prime \prime}$

\footnotetext{
${ }^{7}$ The JCMT is operated by the Joint Astronomy Centre in Hilo, Hawaii, on behalf of the present organizations: the Particle Physics and Astronomy Research Council in the UK, the National Research Council of Canada, and the Netherlands Organization for Scientific Research.
}

Derived Line Parameters for IRAS 16293-2422

\begin{tabular}{ccccc}
\hline \hline Line & $\begin{array}{c}\int T_{\mathrm{mb}} d v \\
\left.(\mathrm{mK} \mathrm{km} \mathrm{s})^{-1}\right)\end{array}$ & $\begin{array}{c}T_{\text {peak }} \\
(\mathrm{mK})\end{array}$ & $\begin{array}{c}\Delta V \\
\left(\mathrm{~km} \mathrm{~s}^{-1}\right)\end{array}$ & $\begin{array}{c}V_{\text {LSR }} \\
\left(\mathrm{km} \mathrm{s}^{-1}\right)\end{array}$ \\
\hline $\mathrm{D}_{2} \mathrm{O} \ldots \ldots$ & $-104(25)$ & $-184(4)$ & $0.53(0.15)$ & $4.09(0.04)$ \\
& $574(75)$ & $88(4)$ & $6.13(0.82)$ & 4.09 \\
\hline
\end{tabular}

Note.-Entries refer to the two-Gaussian components fitted.

with a main-beam efficiency $\left(\eta_{\mathrm{mb}}\right)$ of $63 \%$. We have observed IRAS $16293-2422$ for about $30,000 \mathrm{~s}$, reaching $1 \sigma \mathrm{rms}=$ $37 \mathrm{mK}$ on a single channel. Scans were co-added to produce $T_{A}^{*}$, which was then converted to $T_{\mathrm{mb}}$.

\subsection{The $\mathrm{D}_{2} \mathrm{O}$ Spectrum}

Figure 1 shows the resulting JCMT spectrum after subtraction of the $850 \mathrm{mK}$ continuum level (which was found to be consistent with the background continuum observed by SCUBA). In the same figure, we also display the spectrum of the HDO fundamental transition at $464 \mathrm{GHz}$, obtained with JCMT by Stark et al. (2004) and Parise et al. (2005). The $\mathrm{D}_{2} \mathrm{O}$ and HDO spectra are very similar: they both show a narrow absorption dip at the systemic velocity of IRAS 16293-2422 $\left(\sim 4.0 \mathrm{~km} \mathrm{~s}^{-1}\right)$ superimposed on an emission component whose width is about $6 \mathrm{~km} \mathrm{~s}^{-1}$. In addition, the $\mathrm{D}_{2} \mathrm{O}$ spectrum shows the presence of an emission line at about $10 \mathrm{~km} \mathrm{~s}^{-1}$ (see below). The reality of this line was checked by changing the tuning at a different frequency.

In order to derive the para- $\mathrm{D}_{2} \mathrm{O}$ line-integrated intensity, we fitted the data with a two-component Gaussian model, fixing the line centers of the absorption and emission component according to that found for the HDO line (Stark et al. 2004; Parise et al. 2005). The results are shown in Figure 1 and reported in Table 1. Figure 1 also presents the residuals in the upper portion. One additional spectral component is present around $10 \mathrm{~km} \mathrm{~s}^{-1}$, corresponding to a rest frequency of $316.793 \mathrm{GHz}$, when considering the systemic velocity $\left(\sim 4.0 \mathrm{~km} \mathrm{~s}^{-1}\right)$. We inspected the Jet Propulsion Laboratory spectroscopy database and the Cologne Database for Molecular Spectroscopy (Pickett et al. 1998; Müller et al. 2001) and identified a few candidate species. Ethanol $\left(\mathrm{C}_{2} \mathrm{H}_{5} \mathrm{OH}\right)$ has a line at the right frequency $(316.793460 \mathrm{GHz})$, but the observed signal would imply an implausibly large ethanol column density, and so we discard this possibility. Dimethyl ether $\left(\mathrm{CH}_{3} \mathrm{OCH}_{3}\right)$ and $\mathrm{SO}^{17} \mathrm{O}$, the next most plausible lines, would require velocity shifts of -1.2 and $+3.9 \mathrm{~km} \mathrm{~s}^{-1}$, respectively, from the nominal core $V_{\mathrm{LSR}}$ to match the observed line profile. In addition, the column density of both species would be 10 times larger than those derived by previous observations (Cazaux et al. 2003; Wakelam et al. 2004). We therefore consider these identifications to be highly unlikely. On the other hand, the line intensity of the $\mathrm{CH}_{3} \mathrm{OD}\left(7_{0}-6_{0}+\right)$ transition at $316.795074 \mathrm{GHz}$ (Walsh et al. 2000) is in agreement with the previous detection of this molecule in IRAS 16293-2422 (Parise et al. 2002), and we therefore consider this to be the likely identification.

The presence of the $\mathrm{D}_{2} \mathrm{O}$ line and the line at $10 \mathrm{~km} \mathrm{~s}^{-1}$ are confirmed by earlier lower spectral resolution data obtained with the Caltech Submillimeter Observatory (CSO) $10 \mathrm{~m}$ telescope on 2001 August 1. The spectra were taken in chopping secondary mode with a chop throw of $90^{\prime \prime}$. The back end used was an acusto-optical spectrometer with $500 \mathrm{MHz}$ bandwidth, providing a velocity resolution of $1.33 \mathrm{~km} \mathrm{~s}^{-1}$. The beam efficiency at $316 \mathrm{GHz}$ was measured to be 0.75 , and the beam size is $22^{\prime \prime}$. The system temperature was typically $1000 \mathrm{~K}$, and an rms of $15 \mathrm{mK}$ was reached after 60 minutes of integration. 
The integrated line intensities measured at CSO are about a factor 2 lower than those measured at JCMT; within the calibration uncertainties $(\sim 20 \%)$, this is consistent with the emission of both lines originating in a region of size equal to or smaller than the JCMT beam. Given their higher spectral resolution, only the JCMT measurements are presented here.

In summary, using the velocity parameters of the known HDO emission, we can reproduce both the absorption feature and most of the broad emission centered around the nominal source velocity for the $\mathrm{D}_{2} \mathrm{O}$ line at $316.8 \mathrm{GHz}$. Another feature appears at about $10 \mathrm{~km} \mathrm{~s}^{-1}$ due to $\mathrm{CH}_{3} \mathrm{OD}$.

\subsection{Column Density and $\mathrm{D} / \mathrm{H}$ Ratios}

Observations of singly deuterated water toward IRAS $16293-2422$ show that it is abundant $\left[x(\text { HDO }) \sim 10^{-7}\right]^{8}$ in the inner region of the envelope, where $T_{\text {dust }} \gtrsim 100 \mathrm{~K}$ and ice mantles evaporate (Parise et al. 2005). In this region, the hot corino, the water fractionation ratio in the evaporated ices is estimated to be $\mathrm{HDO} / \mathrm{H}_{2} \mathrm{O} \approx 3 \%$.

The association of the HDO emission with the hot corino is based on a full radiative transfer model of IRAS 16293 (Parise et al. 2005). This considered several HDO lines and employed temperature and density profiles that have been validated by independent observations of the gas and dust emission (Ceccarelli et al. 2000; Schöier et al. 2002). These calculations show that HDO emission from the hot gas dominates because the abundance of HDO is so small $\left[x(\mathrm{HDO}) \leq 10^{-9}\right]$ in the outer region of the envelope, where it is presumably frozen onto the grains. The observed $\mathrm{D}_{2} \mathrm{O}$ line width $\left(\sim 5 \mathrm{~km} \mathrm{~s}^{-1}\right)$ is also consistent with an origin in the hot corino gas. Therefore, it is extremely unlikely that the observed $\mathrm{D}_{2} \mathrm{O}$ line originates in the cold outer envelope and so, in the following, we assume that it also originates in the hot corino.

We assume that the deexcitation collision coefficient for the $\mathrm{D}_{2} \mathrm{O}\left(1_{10}-1_{01}\right)$ transition is equal to $2.9 \times 10^{-10} \mathrm{~s}^{-1}$, the same value as that of the transition from the first excited state to the ground state of ortho- $\mathrm{H}_{2} \mathrm{O}$ resulting from collisions with ortho$\mathrm{H}_{2}$ at a temperature of $100 \mathrm{~K}$ (Green et al. 1993). The corresponding critical density is $\sim 2 \times 10^{6} \mathrm{~cm}^{-3}$. For the physical conditions observed in the hot corino, i.e., a density of $\sim 3 \times$ $10^{7} \mathrm{~cm}^{-3}$ and a temperature of $\sim 100 \mathrm{~K}$ (Ceccarelli et al. 2000) in a region 1.5" in diameter (Kuan et al. 2004; Bottinelli et al. 2004), we can assume that the level populations are likely to be thermalized. This gives a para- $\mathrm{D}_{2} \mathrm{O}$ column density, $N\left(\mathrm{p}-\mathrm{D}_{2} \mathrm{O}\right)$, of $3.5 \times 10^{12} \mathrm{~cm}^{-2}$. Further assuming the thermal equilibrium value $1: 2$ for the $o-\mathrm{D}_{2} \mathrm{O} / \mathrm{p}-\mathrm{D}_{2} \mathrm{O}$ ratio yields the total column of emitting $\mathrm{D}_{2} \mathrm{O}$ molecules: $N\left(\mathrm{D}_{2} \mathrm{O}\right)=1.0 \times 10^{13} \mathrm{~cm}^{-2}$. Adopting $N\left(\mathrm{H}_{2}\right)=6 \times 10^{22} \mathrm{~cm}^{-2}$ (Ceccarelli et al. 2000) gives a $\mathrm{D}_{2} \mathrm{O}$ fractional abundance equal to $1.7 \times 10^{-10}$ and corresponding fractionation ratios of $\mathrm{D}_{2} \mathrm{O} / \mathrm{HDO} \sim 0.0017$ and $\mathrm{D}_{2} \mathrm{O} / \mathrm{H}_{2} \mathrm{O} \sim$ $5 \times 10^{-5}$. As discussed above, the $\mathrm{D}_{2} \mathrm{O}$ line presents a dip of absorption at the systemic velocity of the source, with a measured line-to-continuum ratio equal to about 0.22 and a line width of $0.6 \mathrm{~km} \mathrm{~s}^{-1}$. Assuming that all molecules are in the ground-state level, then $N\left(\mathrm{p}-\mathrm{D}_{2} \mathrm{O}\right) \approx 2.8 \times 10^{12} \mathrm{~cm}^{-2}$ is required to account for this absorption, in good agreement with the column density derived from the emission line. Finally, we emphasize that the observed $\mathrm{D}_{2} \mathrm{O}$ line profile-emission on a $\sim 5 \mathrm{~km} \mathrm{~s}^{-1}$ range with absorption on a $\sim 0.5 \mathrm{~km} \mathrm{~s}^{-1}$ range-is similar to what is observed in many other molecular lines from IRAS 16293, including the HDO line shown in Figure 1. This profile is fully consistent with

\footnotetext{
${ }^{8}$ The value $x$ is the fractional abundance relative to $\mathrm{H}_{2}$.
}

the interpretation that the $\mathrm{D}_{2} \mathrm{O}$ emission originates in the hot corino and is self-absorbed.

\section{DISCUSSION}

The observed $\mathrm{D}_{2} \mathrm{O}, \mathrm{HDO}$, and $\mathrm{H}_{2} \mathrm{O}$ molecules trace mantle material that has recently sublimated from dust grains. Postsublimation, gas-phase processes take $\sim 10^{5} \mathrm{yr}$ to alter the $\mathrm{D} / \mathrm{H}$ ratios in the sublimated species (Rodgers \& Millar 1996). Typical estimates of the chemical ages of hot cores/corinos are lower than this ( $10^{4} \mathrm{yr}$; Charnley et al. 1992) and, for the particular case of the IRAS 16293-2422 hot corino, could be much lower, $\sim 10^{2}-10^{3}$ yr (Wakelam et al. 2005). Therefore, the observed molecular D/H ratios in the hot corinos will be equal to those in the ice and will reflect the formation processes in the preceding cold, prestellar phase. Bergin et al. (1999) showed that the low $\mathrm{HDO} / \mathrm{H}_{2} \mathrm{O}$ ratios seen in massive hot cores $\left(\sim 10^{-4}\right)$ could be understood if interstellar water is formed behind shock waves and condensed as ice. In IRAS 16293-2422, the observed $\mathrm{HDO} / \mathrm{H}_{2} \mathrm{O}$ ratio of 0.03 rules out this possibility (Parise et al. 2005) and, in conjunction with our value for $\mathrm{D}_{2} \mathrm{O} / \mathrm{HDO}$ of $1.7 \times 10^{-3}$, can be used to further constrain the chemical origin of the water.

\subsection{Grain Surface Reactions?}

If water forms via grain surface hydrogenation of $\mathrm{O}$ atoms, then $\mathrm{H}_{2} \mathrm{O}$, HDO, and $\mathrm{D}_{2} \mathrm{O}$ will form contemporaneously, and the abundance ratios will be controlled by the relative accretion rates of $\mathrm{D}$ and $\mathrm{H}$ atoms. This ratio, $r$ say, is dependent only on the gas-phase number densities of $\mathrm{D}$ and $\mathrm{H}$ atoms and is equal to $n(\mathrm{D}) / \sqrt{2} n(\mathrm{H})$. If surface reactions distribute D statistically (cf. Watanabe 2005), then we will have $\mathrm{HDO} / \mathrm{H}_{2} \mathrm{O}$ and $\mathrm{D}_{2} \mathrm{O} / \mathrm{H}_{2} \mathrm{O}$ ratios of $2 r$ and $r^{2}$, respectively. This assumes that the ratio $r$ is constant over time; a more realistic calculation that accounts for the fact that $r$ may vary with time results in the inequality

$$
\left(\frac{\mathrm{D}_{2} \mathrm{O}}{\mathrm{HDO}}\right)_{\text {grain }} \geq \frac{1}{4}\left(\frac{\mathrm{HDO}}{\mathrm{H}_{2} \mathrm{O}}\right)_{\text {grain }} .
$$

Using $\mathrm{HDO} / \mathrm{H}_{2} \mathrm{O} \approx 0.03$ (Parise et al. 2005), equation (1) predicts $\mathrm{D}_{2} \mathrm{O} / \mathrm{HDO}$ greater than $7.5 \times 10^{-3}$, in agreement with the value found in numerical models (0.01; Brown \& Millar 1989) but $\approx 5$ times higher than the observed ratio. It is unlikely that the $\mathrm{D}_{2} \mathrm{O} / \mathrm{HDO}$ ratio is wrong by this factor (the uncertainty in the $\mathrm{H}_{2}$ column density cancels out). On the other hand, our $\mathrm{HDO} / \mathrm{H}_{2} \mathrm{O}$ ratio could be an overestimate if the $\mathrm{H}_{2} \mathrm{O}$ abundance is actually 5 times larger than that reported by Parise et al. (2005). Because the $\mathrm{H}_{2} \mathrm{O}$ lines are optically thick, it is possible that this could be the case (see discussion in Ceccarelli et al. 2000 and in Parise et al. 2005). However, we must conclude that, at present, the published data do not support an origin in grain-surface chemistry.

\subsection{Gas-Phase Ion-Molecule Reactions?}

Water formation via cold ion-molecule chemistry is initiated by proton transfer from $\mathrm{H}_{3}^{+}$to atomic oxygen. $\mathrm{HDO}$ is formed simultaneously with $\mathrm{H}_{2} \mathrm{O}$ via deuteron transfer from $\mathrm{H}_{2} \mathrm{D}^{+}$(as well as $\mathrm{HD}_{2}^{+}$and $\mathrm{D}_{3}^{+}$), and we derive $\mathrm{HDO} / \mathrm{H}_{2} \mathrm{O} \approx \bar{R}$, where $\bar{R}$ is the overall $\mathrm{D} / \mathrm{H}$ ratio in isotopologues of $\mathrm{H}_{3}^{+}$. The $\mathrm{DCO}^{+} / \mathrm{HCO}^{+}$ratio will also be equal to $\bar{R}$, so the $\mathrm{HDO} / \mathrm{H}_{2} \mathrm{O}$ ratio derived by Parise et al. (2005) is consistent with the $\mathrm{DCO}^{+}$/ $\mathrm{HCO}^{+}$ratios seen in a number of dark clouds $(\approx 0.03-0.06$; 
Butner et al. 1995). $\mathrm{D}_{2} \mathrm{O}$, however, is not formed until much later in the chemical evolution of the cloud, via deuteron transfer to the HDO formed earlier, and so the $\mathrm{D}_{2} \mathrm{O} / \mathrm{HDO}$ ratio is a strong function of time, unlike the $\mathrm{HDO} / \mathrm{H}_{2} \mathrm{O}$ ratio, which is always very close to $\bar{R}$. Eventually, a chemical steady state is reached in which $\mathrm{D}_{2} \mathrm{O} / \mathrm{HDO} \approx 0.2 \bar{R}$ (Rodgers \& Charnley 2002), but chemical models show that this can take almost 1 Myr (Roberts \& Millar 2000). Therefore, for ion-molecule chemistry we derive

$$
\left(\frac{\mathrm{D}_{2} \mathrm{O}}{\mathrm{HDO}}\right)_{\mathrm{im}} \leq \frac{1}{5}\left(\frac{\mathrm{HDO}}{\mathrm{H}_{2} \mathrm{O}}\right)_{\mathrm{im}}
$$

Again, using $\mathrm{HDO} / \mathrm{H}_{2} \mathrm{O}=0.03$, we predict a maximum value for the $\mathrm{D}_{2} \mathrm{O} / \mathrm{HDO}$ ratio of 0.006 , consistent with the observed value, which is 3.5 times less. This suggests that the material forming the IRAS 16293-2422 system did not have time to reach a chemical steady state and only underwent dense chemical evolution for a few times $10^{5} \mathrm{yr}$ before the water molecules froze out, as expected in the free-fall collapse of a prestellar core (Brown et al. 1988).

\section{CONCLUSIONS}

We have detected heavy water $\left(\mathrm{D}_{2} \mathrm{O}\right)$ in an astronomical environment for the first time. In IRAS 16293-2422, we determine a total $\mathrm{D}_{2} \mathrm{O}$ column density of $1.0 \times 10^{13} \mathrm{~cm}^{-2}$ and a fractional abundance of $\mathrm{D}_{2} \mathrm{O} / \mathrm{H}_{2}=1.7 \times 10^{-10}$. The derived deuterium fractionation ratios are $\mathrm{D}_{2} \mathrm{O} / \mathrm{HDO}=1.7 \times 10^{-3}$ and $\mathrm{D}_{2} \mathrm{O} / \mathrm{H}_{2} \mathrm{O}=5 \times 10^{-5}$. Shock chemistry can be ruled out as contributing to the origin of the water in this source. At steady state, grain-surface chemistry and ion-molecule reactions both predict $\mathrm{D}_{2} \mathrm{O} / \mathrm{HDO}$ ratios $\approx 4-5$ times higher than observed. However, in the latter case, lower $\mathrm{D}_{2} \mathrm{O} / \mathrm{HDO}$ ratios are possible if the gas-phase chemistry of water and its deuterated isotopologues did not attain a chemical steady state prior to most of the molecules condensing onto dust grains. This scenario is also consistent with the low fractional abundance of water measured in IRAS 16293-2422 and is generally consistent with rapid molecular cloud formation and protostellar evolution (di Francesco et al. 2007; Ceccarelli et al. 2007).

As it is becoming clear that extreme deuteration appears to be common around low-mass protostars (Ceccarelli et al. 2007; Parise et al. 2006), it is likely that the $\mathrm{D}_{2} \mathrm{O} / \mathrm{HDO} / \mathrm{H}_{2} \mathrm{O}$ ratios can be determined in other sources and so shed further light on the chemistry of interstellar isotopic fractionation. Multitransition observations of additional sources are also needed to obtain reliable $\mathrm{H}_{2} \mathrm{O}$ abundances (Maret et al. 2002), and so the Herschel Space Observatory will provide a breakthrough in this respect.

The CSO is funded by the NSF through grant AST 22-09008. This work was supported by the NASA Goddard Center for Astrobiology and, through Cooperative Agreement NCC2-1412, by NASA's Long Term Space Astrophysics Program. J. R. Pardo and J. Cernicharo thank the Spanish MEC for funding support under grant AYA2003-02785 and the Madrid Community Government under grant S-0505 ESP-0237 (ASTROCAM).

\section{REFERENCES}

Bacmann, A., Lefloch, B., Ceccarelli, C., Steinacker, J., Castets, A., \& Loinard, L. 2003, ApJ, 585, L55

Bergin, E. A., Neufeld, D. A., \& Melnick, G. J. 1999, ApJ, 510, L145

Bockelée-Morvan, D., et al. 1998, Icarus, 133, 147

Bottinelli, S., et al. 2004, ApJ, 617, L69

Brown, P. D., Charnley, S. B., \& Millar, T. J. 1988, MNRAS, 231, 409

Brown, P. D., \& Millar, T. J. 1989, MNRAS, 240, 25P

Butner, H. M., Lada, E. A., \& Loren, R. B. 1995, ApJ, 448, 207

Caselli, P., Stantcheva, T., Shalabiea, O., Shematovich, V. I., \& Herbst, E. 2002, Planet. Space Sci., 50, 1257

Cazaux, S., Tielens, A. G. G. M., Ceccarelli, C., Castets, A., Wakelam, V., Caux, E., Parise, B., \& Teyssier, D. 2003, ApJ, 593, L51

Ceccarelli, C. 2002, Planet. Space Sci., 50, 1267

Ceccarelli, C., Castets, A., Caux, E., Hollenbach, D., Loinard, L., Molinari, S., \& Tielens, A. G. G. M. 2000, A\&A, 355, 1129

Ceccarelli, C., \& Dominik, C. 2005, A\&A, 440, 583

Ceccarelli, C., Dominik, C., Caux, E., Lefloch, B., \& Caselli, P. 2005, ApJ, 631, L81

Ceccarelli, C., et al. 2007, in Protostars and Planets V, ed. B. Reipurth, D. Jewitt, \& K. Keil (Tucson: Univ. Arizona Press), 47

Charnley, S. B., Tielens, A. G. G. M., \& Millar, T. J. 1992, ApJ, 399, L71

Charnley, S. B., Tielens, A. G. G. M., \& Rodgers, S. D. 1997, ApJ, 482, L203

di Francesco, J., et al. 2007, in Protostars and Planets V, ed. B. Reipurth, D. Jewitt, \& K. Keil (Tucson: Univ. Arizona Press), 17

Gensheimer, P. D., Mauersberger, R., \& Wilson, T. L. 1996, A\&A, 314, 281 Green, S., Maluendes, S., \& McLean, A. D. 1993, ApJS, 85, 181

Jacq, T., Walmsley, C. M., Henkel, C., Baudry, A., Mauersberger, R., \& Jewell, P. R. 1990, A\&A, 228, 447

Kuan Y.-J., et al. 2004, ApJ, 616, L27

Lis, D. C., et al. 2002, ApJ, 571, L55

Marcelino, N., et al. 2005, ApJ, 620, 308

Maret, S., et al. 2002, A\&A, 395, 573

Meier, R., et al. 1998, Science, 279, 842

Millar, T. J., Bennett, A., \& Herbst, E. 1989, ApJ, 340, 906
Müller, H. S. P., Thorwirth, S., Roth, D. A., \& Winnewisser, G. 2001, A\&A, 370, L49

Nuth, J. A., III, Charnley, S. B., \& Johnson, N. M. 2006, in Meteorites and the Early Solar System II, ed. D. S. Lauretta \& H. Y. McSween, Jr. (Tucson: Univ. Arizona Press), 147

Pardo, J. R., et al. 2001, ApJ, 562, 799

Parise, B., et al. 2002, A\&A, 393, L49

2004, A\&A, 416, 159

2005, A\&A, 431, 547

2006, A\&A, 453, 949

Pickett, H. M., et al. 1998, J. Quant. Spectrosc. Radiat. Transfer, 60, 883

Roberts, H., Herbst, E., \& Millar, T. J. 2003, ApJ, 591, L41

Roberts, H., \& Millar, T. J. 2000, A\&A, 364, 780

Rodgers, S. D., \& Charnley, S. B. 2001, ApJ, 553, 613

. 2002, Planet. Space Sci., 50, 1125

Rodgers, S. D., \& Millar, T. J. 1996, MNRAS, 280, 1046

Roueff, E., \& Gerin, M. 2003, Space Sci. Rev., 106, 61

Schöier, F. L., Jorgensen, J. K., van Dishoeck, E. F., \& Blake, G. A. 2002, A\&A, 390, 1001

Stark, R., et al. 2004, ApJ, 608, 341

Tielens, A. G. G. M. 1983, A\&A, 119, 177

van der Tak, F. F. S., et al. 2002, A\&A, 388, L53

van Dishoeck, E. F., Blake, G. A., Jansen, D. J., \& Groesbeck, T. D. 1995, ApJ, 447, 760

Vastel, C., et al. 2003, ApJ, 593, L97 . 2004, ApJ, 606, L127

Wakelam, V., Selsis, F., Herbst, E., \& Caselli, P. 2005, A\&A, 444, 883

Wakelam, V., et al. 2004, A\&A, 422, 159

Walsh, M. S., et al. 2000, J. Mol. Spectrosc., 204, 60

Watanabe, N. 2005, in IAU Symp. 231, Astrochemistry: Recent Successes and Current Challenges, ed. D. C. Lis, G. A. Blake, \& E. Herbst (Cambridge: Cambridge Univ. Press), 415

Watson, W. D. 1976, Rev. Mod. Phys., 48, 513 